Z. klin. Chem. u. klin. Biochem.

7. Jg., S. 66-71, Januar 1969

\title{
Erfahrungen mit dem Technicon SMA 12
}

\author{
Von K. Schily, M. Eggstein, W. Knodel, R. Allner, S. Henig und E. Kuhlmann \\ Aus der Mediqinischen Universitätsklinik Tübingen (Direktor: Prof. Dr. H. E. Bock)
}

(Eingegangen am 15. November 1968)

Herrn Prof. Dr. H. E. Bock qum 65. Geburtstag gewidmet

\begin{abstract}
Das Analysengerät SMA 12 Typ „,Survey“ liefert unter Routinebedingungen Cholesterin-, Harnstoff-, Gesamteiweiß-, Albumin-, Calcium-, Phosphor- und Bilirubinwerte, die bei serieller Messung mit einem Variationskoeffizienten $<2 \%$ belastet sind. Für Kreatinin beträgt der Variationskoeffizient 2,7\%, für Harnsäure 3,9\%. Daraus berechnet sich ein Wiederholstreubereich für Serumanalysen im SMA 12 Enzyme ausgenommen - zwischen 7-10\% für Kreatinin und Harnsäure und 5\% für die übrigen Messungen.

Die Tag-zu-Tag-Präzision von Cholesterin, Harnstoff, Harnsäure, Eiweiß, Albumin, Calcium und Phosphor im SMA12 charakterisieren Variationskoeffizienten $<3,9 \%$. Für Kreatinin betragen sie zwischen 4,2 und 9,0\%, für Bilirubin zwischen 1,6-6,7\%. Die Vergleichsstreubereiche sind über 2-3 Wochen am Standard- und Poolserum gemessen für die meisten SMA12-Analysen mit $\leqq 10 \%$, für Kreatinin mit $13 \%$ anzusetzen.

Ausreichende Einarbeitung, sorgfältige Wartung und Bedienung des SMA12-Gerätes und die Qualität bzw. Stabilität der Kontroll- bzw. Standardsera wirken sich trotz vollmechanisierter Analysentechnik entscheidend auf die Präzision der Analysen aus. Sie ist im präparierten, laboreigenen Standard nicht schlechter als in fertigkäuflichen Standardsera, wird in 7 von 9 Analysenkanälen durch bevorzugte Gerätebedienung und -wartung signifikant verbessert und liegt für einige Analysen besser, für andere so gut wie die Präzision in der Serie und von Tag zu Tag von manuellen bzw. 1-bis 4-Kanal-Autoanalyzermessungen.

Im SMA12 bestimmte Enzymaktivitäten lassen sich aus bekannten Gründen nicht mit den im optischen Test gemessenen vergleichen. Im Aspartat-Transaminase-Kanal messen wir deshalb - nach entsprechendem Umbau - Kreatinin, die Kanäle für alkalische Phosphatase und Lactatdehydrogenase bleiben ungenutzt. Das 12-Kanalsystem wird zu einer 10-Kanal-Meßeinheit. Sie garantiert mit weniger aufwendigem Reagenzienbedarf Werte für Glucose, Cholesterin, Kreatinin, Harnstoff, Harnsäure, Bilirubin und (mit Vorbehạlt) Werte für Gesamteiweiß, Albumin, Calcium und Phosphor, die sich nicht von denen mit konventioneller Technik, im Notdienst und bei Systemausfall gewonnenen Werten unterscheiden.
\end{abstract}

\section{Observations on the use of the Technicon SMA 12}

The analyzer SMA 12 type „Survey“ under routine conditions determines values for cholesterol, urea, total protein, albumin, calcium, phosphorus and bilirubin, which, in serial measurement, are subject to $\mathrm{V}<2 \%$, for creatinine to $2.7 \%$, for uric acid $3.9 \%$. In the SMA 12 , the replicate scatter for serum analyses (enzymes excepted) lies between 7 and $10 \%$ for creatinine and uric acid and is $5 \%$ for the other measurements.

The day to day precision for cholesterol, urea, uric acid, protein, albumin, calcium and phosphorus in the SMA 12 is characterised by variation coefficients of $3.0 \%$ or less. For creatinine they lie between 4.2 and $9.0 \%$, for bilirubin between 1.6 and $6.7 \%$. Over a period of $2-3$ weeks, most measurements on standard and pool serum in the SMA 12 show a replicate scatter of $10 \%$ or less, with $13 \%$ for creatinine.

Despite the fully mechanised analytical technique, the precision of the analyses are affected by appropriate training, careful servicing and maintenance of the SMA 12 apparatus, and by the quality and stability of the control and standard sera. Prepared laboratory standards give results equal to those with ready made commercial standards. Significant improvement is obtained in 7 out of the 9 analytical channels by special servicing and maintenance of the apparatus. The precision of some analyses is better and for others it is equal to that of serial and day to day manual analyses, or 1 to 4 channel autoanalyzer measurements.

It is clear that enzyme activities measured in the SMA 12 cannot be compared with those measured optically. Therefore, following an appropriate conversion, we measured creatinine in the aspartate transaminase channel, while the channels for alkaline phosphatase and lactate dehydrogenase remained unused. The 12-channel system is used as a 10-channel measuring unit. The consumption of reagents is economical, and it guarantees values for glucose, cholesterol, creatinine, urea, uric acid, bilirubin and (with certain provision) values for total protein, albumin, calcium and phosphorus, which do not differ from those obtained by conventional methods, in the emergency routine, or in systemic failure.

Mit dem Technicon SMA 12 (Sequential Multiple Analyzer) Typ „Survey“ lassen sich Cholesterin, Calcium, Phosphor, Bilirubin, Gesamteiweiß, Albumin, Harnsäure, Harnstoff, Glucose, Lactatdehydrogenase, alkalische Phosphatase und Aspartat-Transaminase in $3,0-3,2 \mathrm{~m} l$ Blutserum bestimmen. Die Kapazität beträgt 30 Proben pro Std., Eich- und Kontrollsera eingerechnet.

Das Analysensystem ist starr, Modifikationen - sofern überhaupt möglich —, verlangen Änderungen des Schlauchsystems und Eingriffe in die elektronischen Bauelemente.

1-, 2- bis 4-Kanal-Autoanalyzer mit sequentiellem oder simultanem Analysengang empfehlen wir, wenn für eine bestimmte Untersuchung mehr als 40 Proben täglich anfallen. Das 12fach Analysengerät Typ „Survey“ läuft mit 40 Analysen pro Tag knapp 2 Stdn. Die mit dem Betrieb eines derartigen Apparates verbundene Organisation (Einarbeitung von Bedienungspersonal, . Wartungsleistungen, Reagenzienbeschaffung, Prüfverfahren) scheint uns erst $\mathrm{ab}$ wenigstens 80 bis 120 Proben pro Tag angezeigt.

Simultan-Analysengeräte aktualisieren das Problem „indiskriminierte klinisch-chemische Diagnostik" oder „klinisch-chemischer check up“, anstelle bisher üblicher „ausgewählter, indizierter" Anforderungen von klinisch-chemischen Parametern. Hieraus erwachsen neuartige Wertungsmaßstäbe im ärztlichen, diagnostischen 
Bereich (1-3), in der Rentabilitätsbeurteilung von Analysengeräten und in der Kalkulation klinisch-chemischer Analysenspektren. Diesen Erörterungen geht jedoch die Frage nach der Zuvcrlässigkeit und Verglcichbarkeit von SMA12-Resultaten voraus.

\section{Methodische Angaben}

In der Einarbeitungsphase wurden zunächst dic Standardausführung des SMA12, Typ „Survey“, dic vom Hersteller empfohlenen Flußdiagramme und Nachweisteaktionen, Reagenzien und Standardscra verwendet. Nach etwa dreimonatiger Routine haben wir dic Schlauchsysteme des SMA12 weitgehend durch Glastohre crsetzt. Der Aspartat-Transaminasc-Kanal wurde von der Lieferfirma für den Nachwcis von Kreatinin umgebaut.

Sämtliche Messungen bcim SMA12 Typ „Survcy" crfolgen kolorimetrisch und werden auf ein von der Herstellerfirma geliefertes Standardscrum bezogen. Wechsclscitige Probencinfluisse werden durch dic zwischengeschalteten Spülungen weitgehend verhindert. Dic Justicrung erfolgt nach den Empfehlungen des Herstellers. Dic Gerätceinstellung, "phasing“- und Driftkorrckturen verlangen das empfohlene bzw. cin erweitertes Beschickungsschema.

Seit Abschluß der hier mitgetcilten Prüfungen beachten wir folgendes Einstell-, Kontroll- und Beschickungsschema: in den Probenteller kommen zunächst cine Leerprobe für dic $100 \%$-Transmissions-Kontrolle, 5 Proben mit belicbigem oder getestetem Poolscrum zur phasing-Kontrolle, dann einc Lcerprobc, 2 wäßr. Krcatininstandards und 2 frischangesetzte Standards zur Eichung der cinzelnen Mcßkanäle des SMA12, danach 2 Proben mit Standard vom Vortag und cinc Probe getestetes Poolserum zur Präzisionskontrollc. Jetzt folgen Patientensera. Jede 10. Probe cnthält getestetes Poolscrum, die 20. und 21. Wasser, dic 22. und 23. Standard. Dann kommen wieder Sera. Evtl. erforderliche Nacheichungen werden beim Durchlauf der 2. Wasser- bzw. 2. Standardprobe (also nicht während einer unmittelbar auf Serum folgenden Lcerprobc und nicht während des direkt auf Wasser folgenden Standards) vorgenommen.

Diese Empfehlung sollte nicht starr übcrnommen, sic muß periodisch überprüft und variiert werden.

Gesamicbolesterin wird mit der Liebermann-Burchard-Reaktion in der von HuANG und Mitarbeitern (4) beschriebenen Variation (ohne Dialyse, $37^{\circ}$ ) bestimmt. Als Reagenz dient ein Gemisch aus Eisessig, Essigsäurcanhydrid und konz. Schwefelsäure. Der cmpfohlenc Zusatz von wasserfreiem Natriumsulfat führt zu Verstopfungen im Schlauchsystem. Wir haben das $\mathrm{Na}_{2} \mathrm{SO}_{4}$ weggelassen, setzen aber das Reagenz täglich frisch an. Kolorimetriert wird bei $630 \mathrm{~nm}$. Bilirubinwcrtc über $1,5 \mathrm{mg} / 100 \mathrm{~m} /$ stören. Dcr Fehler soll sich korrigieren lassen, für jedes $\mathrm{mg}$ Bilirubin übcr $1,5 \mathrm{mg}$ (bezogen auf $100 \mathrm{~m} / \mathrm{Scrum}$ ) werden vom Cholesterinwert $10 \mathrm{mg} / 100 \mathrm{~m} /$ abgezogen.

Calcium und anorganisches Plosphat werden aus dem angesäuerten Scrum bei $37^{\circ}$ abdialysiert und Calcium als Farbkomplex mit Kresolphthalcin-Komplexon bei $580 \mathrm{~nm}$ bestimmt (5).

Phosphat wird als Phosphormolybdänblau nach Reduktion mit Zinn-II-chlorid bei $660 \mathrm{~nm}$ gemessen.

Gesamtbilirubin reagiert in Coffein-Natriumbenzoat-Lösung mit diazotierter Sulfanilsäure, dic Farbintensität des Azofarbstoffes wird - ohne Dialyse - bei $600 \mathrm{~nm}$ mit der des Standards verglichen (6).

Der Albuminnaclsweis beruht auf dem "selektiven" Farbstoffbindevermögen des Albumins mit 2-(4-Hydroxyazobenzol)-benzocsäure. Geeicht wird gegen SMA 12 Standard bei $505 \mathrm{~nm}$ (7).

Für Gesanteinveiß dient cine modifizicrte Biuretreaktion, photometriert wird bei $550 \mathrm{~nm}(8)$.

Harnsäure dialysiert im SMA12 gegen $\mathrm{NaCl}$-Lösung, wird alkalisicrt und reduziert bei Raumtempcratur zweiwertiges Kupfer. Ge- bildctes Kupfer-I wird mit Bathocuproin bei $486 \mathrm{~nm}$ nachgewiesen. Glucose, Sulfhydrylgruppen, Ascorbinsäure und Kreatinin sollen unter den angegebenen Reaktionsbedingungen nicht stïren (9).

Harnsıoff reagiert nach Dialyse in saurem Milicu bei $95^{\circ}$ mit Diacctylmonoxim in $\mathrm{Gegenwart}$ von $\mathrm{FeCl}_{3}$ und Thiosemicarbazid. Dicses intensivicrt dic Farbbildung und ersetzt konzentricrte Säuren. Das gefärbtc Reaktionsprodukt wird bei $520 \mathrm{~nm}$ gemcssen (10).

Glucose dialysicrt gegen Kochsalz und reduziert nach $\mathrm{Na}_{4} \mathrm{CO}_{3}$ Zugabe unter den im SMA12 gegebenen Bedingungen (Inkubation bei $95^{\circ}$ ) Kupfer-II zu Kupfer-I, welches mit Ncocuproin bei $460 \mathrm{~nm}$ nachgewiesen wird. Anderc reduzicrende Substanzen wic Kreatinin oder Harnsäurc kommen im Scrumdialysat normalerweise nur in so geringen Konzentrationen vor, daß hicrdurch bedingte Störungen vernachlässigt werden können. Durch das zugemischte Natriumcarbonat sollen Ascorbinsäure und Sulfhydrylgruppen weitgehend climiniert werden.

Alkalisclue Phospljatase: Nach W. MarsH und Mitarbeiter (11) wird cine Modifikation der King- $\Lambda$ rmstrong-Mcthode durchgeführt. $\Lambda$ us Phenylphosphat durch alkalische Phosphatase freigesetztes Phenol wird mit 4-Aminoantipyrin kondensicrt und mit Ferricyanid zu einem roten Farbstoff oxydiert, der bei $505 \mathrm{mn}$ gemessen wird. Wertangabe in King-Armstrong-Einheiten (37 $)$.

Bei der durch Lactatdelyydrogenase katalysierten Oxydation von Lactat zu Pyruvat entstcht NADH, dieses reduzicrt mit Diaphorasc versetztes 3-p-Nitrophenyl-1,2-p-Jodophenyl-5-Phenyltetrazoliumchlorid zu cinem purpurroten Formazan $(505 \mathrm{~nm})(12,13)$. Aktivitätsmaß in WACKrR-Einhciten. Eincr Einheit entspricht cinc Extinktionsdifferenz $(340 \mathrm{~nm}, \mathrm{~d}=1 \mathrm{~cm})$ von 0,001 pro $\mathrm{Min}$. im Endvolumen von $3,0 \mathrm{~m} /(14)$.

Durch Aspartat-Transaminase aus Aspartat entstandenes Oxalacetat wird gegen Citratpuffer dialysiert und an Azoene Fast Red gekuppelt, bei $460 \mathrm{~nm}$ photometriert (15). Resultat in KARMEN-Einheiten. Einer Einheit entspricht einc Extinktionsdifferenz $(340 \mathrm{~nm}$, $\mathrm{d}=1 \mathrm{~cm}$ ) von 0,001 pro Min. bei $37^{\circ} \mathrm{im}$ Endvolumen von $2,8 \mathrm{~m} /$ (16).

Kreatinin reagicrt im Scrumdialysat mit Pikrinsäurc und $\mathrm{NaOH}$ (JAFre'sche Reaktion) und wird bei $505 \mathrm{~nm}$ photometricrt.

\section{Fragestellung und Auswertung}

Die Versuche galten

1. der Wiederbolbarkeit (Präzision in der Serie) von Serumanalysen (Tab. 1 und 2) und Standardsera (Tab. 3),

a) in der Einarbeitungszeit,

b) in der Betriebsphase, verglichen mit

c) der Wiederholbarkeit bisher üblicher Serumanalysen (Tab. 1 und 2).

2. der Reproduzierbarkeit (Präzision von Tag zu Tag) von Serumanalysen ( $\mathrm{Tab} .4$ und 5 ) und Standardsera (Tab. 6) in der Betriebsphase des SMA 12. Die Gerätebedienung erfolgte

a) durch eine erfahrene, geschulte $\dot{\mathrm{M} T A}$,

b) durch eine eingearbeitete, aber mit Laborarbeit zusätzlich belastete MTA.

c) Zum Vergleich werden die Vergleichsbereiche der bisherigen Methoden (Tab. 4 und 5) angegeben.

3. dem Vergleicls von SMA12-Werten mit bisherigen und im Notdienst ferner üblichen manuellen Meßverfahren in Blutproben von nicht ausgewählten Patienten (Vergleiche von Stichproben - Tab. 7 und 8). 
Tab. 1

Mittelwerte und Standardabweichungen für Serumuntersuchungen in der Serie mit dem SMA 12 (Grundwerte für den Wiederholstreubereich $[n=15]$ für SMA 12-Analysen in Sammelserum $[P])$
$\mathrm{HM}=$ manuelle Analysentechnik $\mathrm{AA}=\mathrm{im}$ 1- oder 4-Kanal-Autoanalyzer bestimmt

\begin{tabular}{|c|c|c|c|c|c|c|c|c|c|c|}
\hline \multirow[b]{2}{*}{$\begin{array}{l}\text { Cholesterin } \\
\text { Calcium }\end{array}$} & \multirow[b]{2}{*}{$\underset{\mathrm{mgVal} / \mathrm{l}}{\mathrm{mg} / 100 \mathrm{ml}}$} & \multicolumn{2}{|c|}{$\begin{array}{c}\text { a) Einarbeitungszeit } \\
\overline{\bar{x}} \pm \sigma \\
\left(\mathrm{P}^{\mathrm{a}}\right) \\
\end{array}$} & \multicolumn{3}{|c|}{ b) Betriebsphase $\left(\mathrm{P}_{\overline{\mathrm{x}} \pm \sigma}^{\mathrm{b}} \mathrm{V}_{\%}\right.$} & \multicolumn{3}{|c|}{ c) mit bisheriger Analysentechnik } & \multirow{2}{*}{$\frac{\mathrm{n}}{20}$} \\
\hline & & $\begin{aligned} 225,6 & \pm 4,2 \\
3,9 & \pm 0,175\end{aligned}$ & $\begin{array}{l}1,9 \\
4,5\end{array}$ & $\begin{array}{r}226,1 \\
6,54\end{array}$ & $\begin{array}{l} \pm 3,8 \\
\pm 0,05\end{array}$ & $\begin{array}{l}1,7 \\
0,8\end{array}$ & $\begin{array}{r}210,5 \\
4,3 \\
4,1\end{array}$ & $\begin{array}{l} \pm 3,5(\mathrm{HM}) \\
\pm 0,18(\mathrm{HM})\end{array}$ & $\begin{array}{c}1,7 \\
4,2-7,3\end{array}$ & \\
\hline Phosphat & $\mathrm{mg} / 100 \mathrm{ml}$ & $5,2 \pm 0,24$ & 4,6 & 4,44 & $\pm 0,05$ & 1,1 & $\begin{array}{l}\mathbf{4}, 1 \\
\mathbf{6}, 3 \\
6,5\end{array}$ & $\begin{array}{l} \pm 0,14(\mathrm{AA}) \\
\pm 0,28(\mathrm{HM})\end{array}$ & $2,6-4,3$ & $\begin{array}{l}10 \\
10\end{array}$ \\
\hline $\begin{array}{l}\text { Bilirubin } \\
\text { Albumin } \\
\text { Gesamt-Eiweiß } \\
\text { Harnsäure }\end{array}$ & $\begin{array}{r}\mathrm{mg} / 100 \mathrm{ml} \\
\mathrm{g} / 100 \mathrm{ml} \\
\mathrm{g} / 100 \mathrm{ml} \\
\mathrm{mg} / 100 \mathrm{ml}\end{array}$ & $\begin{array}{l}0,78 \pm 0,04 \\
3,97 \pm 0,06 \\
7,49 \pm 0,12 \\
7,06 \pm 0,47\end{array}$ & $\begin{array}{l}5,1 \\
1,5 \\
1,6 \\
6,7\end{array}$ & $\begin{array}{l}0,7 \\
4,01 \\
7,5 \\
7,22\end{array}$ & $\begin{array}{l} \pm 0 \\
\pm 0,06 \\
\pm 0,11 \\
\pm 0,28\end{array}$ & $\begin{array}{l}0 \\
1,5 \\
1,5 \\
3,9\end{array}$ & $\begin{array}{l} \\
\\
7,62 \\
5,5 \\
5,17\end{array}$ & $\begin{array}{l} \pm 0,00(\mathrm{MM}) \\
\pm 0,04(\mathrm{HM}) \\
\pm 0,12 \text { (AA) }\end{array}$ & $\begin{array}{l}- \\
0,5 \\
2,2\end{array}$ & $\frac{10}{15}$ \\
\hline Harnstoff & $\mathrm{mg} / 100 \mathrm{ml}$ & $\pm 0,645$ & 1,0 & 72,8 & $\pm 0,56$ & 0,8 & $\begin{array}{l}64,4 \\
69\end{array}$ & $\begin{array}{l} \pm 2,0 \\
+2,6\end{array}$ & 3,1 & $\begin{array}{l}10 \\
10\end{array}$ \\
\hline $\begin{array}{l}\text { Glucose } \\
\text { Lactatdehydrogen } \\
\text { Alk. Phosphatase } \\
\text { Aspartat-Transam } \\
\text { Kreatinin }\end{array}$ & $\begin{array}{c}\mathrm{mg} / 100 \mathrm{ml} \\
\text { nase } \mathrm{WE} \\
\mathrm{K}-\mathrm{A}-\mathrm{E} \\
\text { minase } \mathrm{KE} \\
\mathrm{mg} / 100 \mathrm{ml}\end{array}$ & $\begin{array}{r}173,3 \\
122,3 \\
11,8 \\
13,9 \\
25,5 \\
\pm 2,3 \\
\end{array}$ & $\begin{array}{r}2,8 \\
10,9 \\
9,3 \\
8,6 \\
\end{array}$ & 3,3 & $\begin{array}{l}\bar{z} \\
\bar{z} \\
\pm 0,09\end{array}$ & $\begin{array}{l}\bar{z} \\
\overline{2,7}\end{array}$ & $\begin{array}{r}93 \\
\text { IE } 101,6 \\
\text { IE } 23,0 \\
\text { IE } 9,5 \\
2,6 \\
2,6 \\
2,6\end{array}$ & $\begin{array}{l} \pm 4,7 \text { (AA) } \\
\pm 8,6(\mathrm{HM}) \\
\pm 1,04(\mathrm{HM}) \\
\pm 1,87(\mathrm{HM}) \\
\pm 0,1(\mathrm{HM}) \\
\pm 0,06(\mathrm{AA})\end{array}$ & $\begin{array}{r}5,1 \\
8,5 \\
4,5 \\
19,7 \\
3,8 \\
2,3\end{array}$ & $\begin{array}{l}10 \\
50 \\
12 \\
50 \\
10 \\
10\end{array}$ \\
\hline
\end{tabular}

4. dem Vergleich der in Standardsera und Poolserum erreichten Präzision

a) in der Serie und

b) von Tag zu Tag (Tab. 9).

Die Prüfungen erstreckten sich zunächst auf die 12

Original-SMA12-Kanäle. In der jetzigen Betriebsphase beschränken wir uns auf 9 bzw. 10 Serumanalysen:

$\begin{array}{ll}\text { Kreatinin } & \text { Bilirubin } \\ \text { Harnstoff } & \text { Cholesterin } \\ \text { Harnsäure } & \text { Calcium } \\ \text { Gesamteiweiß } & \text { Phosphor } \\ \text { Albumin } & \text { Glucose }\end{array}$

Die Glucosebestimmung im SMA12 wird (aus organisatorischen Gründen) als „orientierende Information" gewertet.
Tab. 2

F-Prüfung der Wiederholstreubereiche (S) für SMA 12 Analysen $(n=15)$ im Sammelserum a) bei Inbetriebnahme, b) nach ausreichenverfahren

$$
<; \geqq \begin{aligned}
& \text { F-Prüfung signifikant }(P=99 \%) \\
& \leqq \text { F-Prüfung signifikant }(P=95 \%)
\end{aligned}
$$

\begin{tabular}{|c|c|c|c|}
\hline Wiederholstreubereich für & $a$ und $c$ & $b$ und $c$ & $a$ und $b$ \\
\hline $\begin{array}{l}\text { Cholesterin } \\
\text { Calcium } \\
\text { Phosphat }\end{array}$ & $\begin{array}{l}a=c(H M) \\
a=c \\
a=c(A A) \\
a=c(H M)\end{array}$ & $\begin{array}{l}\mathrm{b}=\mathrm{c}(\mathrm{HM}) \\
\mathrm{b}<\mathrm{c} \\
\mathrm{b}<\mathrm{c}(\mathrm{AA}) \\
\mathrm{b}<\mathrm{c}(\mathrm{HM})\end{array}$ & $\begin{array}{l}a=b \\
a>b \\
a>b\end{array}$ \\
\hline $\begin{array}{l}\text { Bilirubin } \\
\text { Albumin } \\
\text { Gesamt-Eiweiß } \\
\text { Harnsäure }\end{array}$ & $\begin{array}{l}- \\
a>c(H M) \\
>c(A A)\end{array}$ & $\begin{array}{l}\overline{ } \\
b>c(H M) \\
b . \geq c(A A)\end{array}$ & $\begin{array}{l}a>b \\
a=b \\
a=b \\
a=b\end{array}$ \\
\hline Harnstoff & $a=c(A M)$ & $b<c$ (AA) & $a=b$ \\
\hline $\begin{array}{l}\text { Glucose } \\
\text { Lactatdehydrogenase } \\
\text { alkal. Phosphatase } \\
\text { Aspartat-Transaminase } \\
\text { Kreatinin }\end{array}$ & $\begin{array}{l}a=c(A A) \\
a=c \\
a=c \\
a=c\end{array}$ & $\begin{array}{l}= \\
= \\
b=c(A A) \\
b=c(H M)\end{array}$ & E \\
\hline
\end{tabular}

Tab. 3

Mittelwerte und Standardabweichungen für "Standard"-Untersuchungen in der Serie im SMA 12 (Grundwerte für den Wiederholstreubereich für SMA 12-Analysen [n=15] in Standardsera [St $t_{1} u$. S $t_{2}$ ])

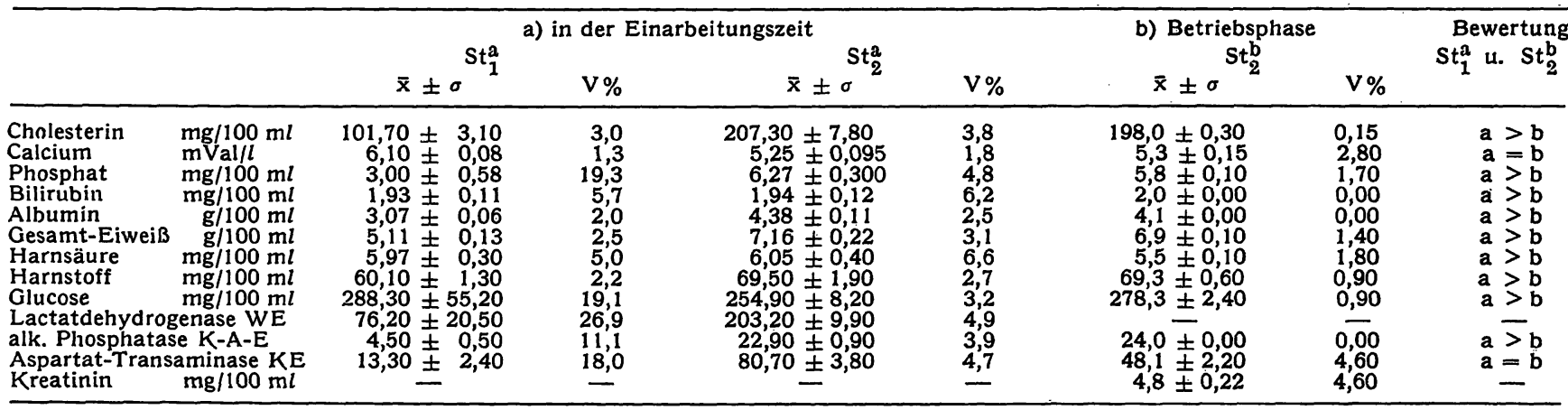

Tab. 4

Mittelwerte und Standardabweichungen für Serumanalysen an verschiedenen Tagen im SMA 12 von 2 unterschiedlich belasteten MTA gemessen (Grundwerte für die Präzision von Tag zu Tag für SMA 12-Analysen im Sammelserum [P])

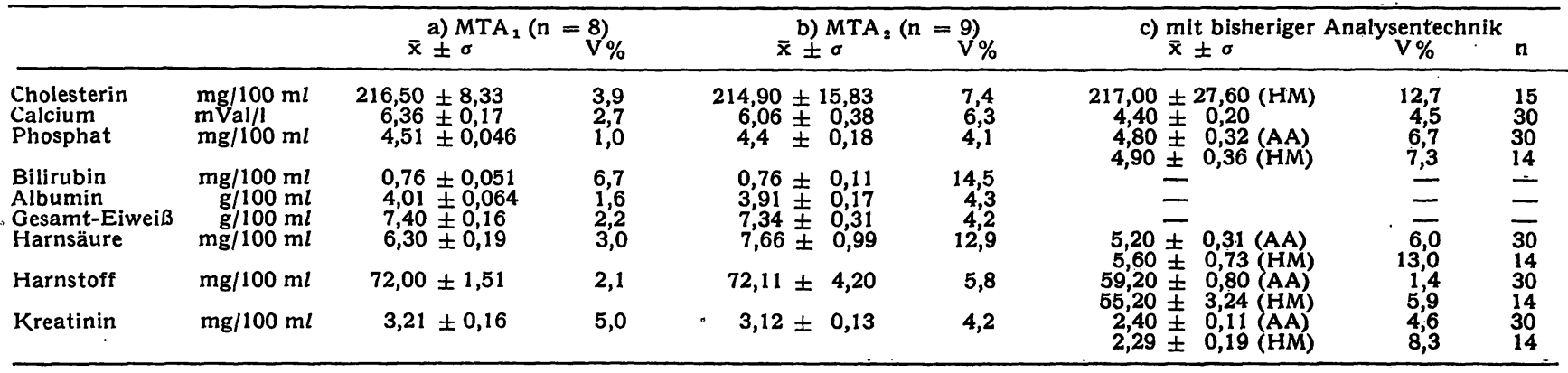


Für die Versuche wurden Poolscrum und 2 Standardscra verwendet, für den Vergleich - „SMA12-Resultate" und „Ergelonisse manucller bzw. 1-, 2- oder 4-KanalAutoanalyzerbestimmungen" - unausgewählte $\mathrm{Pa}$ tientensera. Dic Resultate der ersten Wochen - der „Einarbeitungsphasc" - werden späteren, aus dem Routinclsetricb - der „Betricbsphase“ - gegenübergestellt und mit der Präzision bisher üblicher Verfahren verglichen (3).

Dic Wiederbolbarkeit, - Präzision in der Scrie, repentability (17), der Wiederholstreubereich - wurde aus 15 in unmittclbarer Folge analysierten Proben, die Reprodurierbarkeit - Präzision von Tag zu Tag, rcproducibility (17), der Verglcichsstrcubereich (DIN 15849) aus 8 bis 15 übcr 2 bis 3 Wochen verstreuten Einzelanalysen in Standardseren und Poolserum bercehnet. Als Kriterium dient dic Standardabweichung bəw. der daraus bercchnete Streubereich und der Variationskoeffizient.

Tab. 5

F-Prafung der Tag zu Tag Streuung bel SMA12-Analysen lin Sammelserum

a) Betricbsphase mit MTA 1 (P')

c) "Konventionelle" Meßverfahren

\begin{tabular}{|c|c|c|c|}
\hline $\begin{array}{l}\text { Streuung von } \\
\text { Tag zu Tag für }\end{array}$ & $a$ und $c$ & b und $c$ & $a$ und $b$ \\
\hline $\begin{array}{l}\text { Cholesterln } \\
\text { Calclunı }\end{array}$ & $\begin{array}{l}a<c(H M) \\
a=c\end{array}$ & $\begin{array}{l}b=c \\
b>c\end{array}$ & $\begin{array}{l}a<b \\
a<b\end{array}$ \\
\hline Phosphor & $a<c\left(\begin{array}{l}A A) \\
(H M)\end{array}\right.$ & $b=c$ & $a<b$ \\
\hline $\begin{array}{l}\text { Bllirubin } \\
\text { Albumin } \\
\text { Ocsami-Eiweli } \\
\text { Harnsäure }\end{array}$ & $\overline{-}$ & $\bar{z}$ & $\begin{array}{l}a<b \\
a \leq b \\
a \leq b\end{array}$ \\
\hline Harnstoff & $\begin{array}{l}a=c(A A) \\
a<c(H M)\end{array}$ & $\begin{array}{l}b>c(A A) \\
b=c(H M)\end{array}$ & $a<b$ \\
\hline Kreatinin & $a=c(\stackrel{(A A)}{H M})$ & $b=c\left(\begin{array}{l}A A) \\
H I M)\end{array}\right.$ & $a=b$ \\
\hline
\end{tabular}

Vergleiche basicren auf dem Verhältnis der Varianzen ( $F-$ Prüfung, vgl. l. c. (18)) oder auf der Prüfung der Signifikanz der Diffcrenz zwcicr Mittclwerte.

\section{Resultate und Diskussion}

Dic Resultate sind in den Tabcllen 1-9 zusammengestcllt.

\section{Wiedcrholbarkeit}

a) Während der „Einarbcitung“ beträgt der Variationskoeffizient $\mathrm{V}$ für cinc unter identiscljen Bedingungen wiederholte Cholesterin-, Harnstoff-, Gesamtciwciß- und Albuminmessungen im Serum (Tab. 1, a) weniger als $2 \%$, für Glucosc knapp $3 \%$, für Calcium, Phosphor, Bilirubin und Harnsäure zwischen 4,5 und 6,7\%. Die Enzymaktivitätsmessungen weisen noch größcrc Standardabweichungen auf.

b) Mit längerem Einsatz des SMA12 lassen sich dic Calcium-, Phosphor- und Bilirubinbestimmungen (Tab. 1, b, Tab. 2, a/b) signifikant verbessern. Schließlich werden für die „turnusmäßig“ ermittclten V der Ċholesterin-, Harnstoff-, Gcsamtciwciß-, Albumin-, Phosphor-, Calcium- und Bilirubinbestimmungen im Poolscrum weniger als $2 \%$, für Kreatinin $2,7 \%$ und Harnsüurc 3,9\% berechnet (Tab. 1, b).

$\Lambda$ uch für Untersuchungen in Standardsera konnte dic Präzision in der Seric bis zur Betriebsphase ('Tab. 3) so verbessert werden, daß nur noch die Aspartattransaminase-, Kreatinin- und Calciumwicderholungen mit eincm V über $2 \%$ bclastet sind (Tab. $3, \mathrm{~b}$ ).

c) Beim Vergleich mit den "konventioncllen" Meßverfahren schneiden Calcium, Phosphor und Harnstoff

Tab. 6

Miltelwerte und Standardabweichungen für Standardsera an verschiedenen Tagen Im SMA 12 in verschiedenen Betriebspliasen gemessen

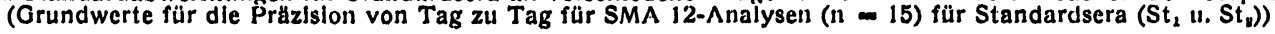

\begin{tabular}{|c|c|c|c|c|c|c|c|c|c|c|}
\hline \multirow{3}{*}{\multicolumn{2}{|c|}{ 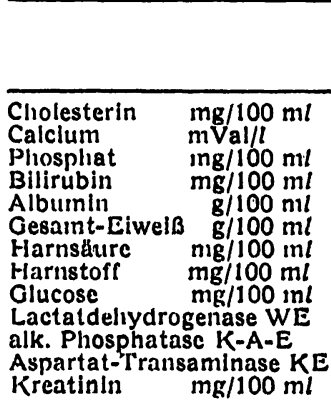 }} & \multicolumn{3}{|c|}{ a) In der Elnarbeitungszelt } & \multirow[b]{2}{*}{$V \%$} & \multicolumn{4}{|c|}{ b) Betricbsphase } & \multirow{2}{*}{$\begin{array}{c}\text { Bewertung } \\
\text { St }{ }_{1}^{a} \text { u. St }\end{array}$} \\
\hline & & $\mathrm{x} \pm \sigma^{\mathrm{St}_{1}^{\mathrm{a}}}$ & $\mathrm{V} \%$ & $z \pm \sigma^{S t a}$ & & $\bar{x} \pm$ & $\sigma \quad \mathrm{St}_{\mathbf{3}}^{\mathrm{D}}$ & $V \%$ & $n$ & \\
\hline & & $\begin{array}{r}111,60 \pm 25,00 \\
4,04 \pm 1,40 \\
3,26 \pm 1,14 \\
1,95 \pm 0,14 \\
2,92 \pm 0,20 \\
5,13 \pm 0,37 \\
5,76 \pm 0,77 \\
111,50 \pm 33,30 \\
305,70 \pm 17,90 \\
79,70 \pm 43,40 \\
11,50 \pm 5,30 \\
8,10 \pm 8,30\end{array}$ & $\begin{array}{r}22,4 \\
34,7 \\
35,0 \\
7,2 \\
6,8 \\
7,2 \\
13,4 \\
29,9 \\
5,9 \\
54,5 \\
46,1 \\
102,5 \\
\end{array}$ & $\begin{array}{r}199,80 \pm 0,30 \\
3,33 \pm 1,23 \\
5,79 \pm 1,91 \\
1,91 \pm 0,12 \\
4,21 \pm 0,13 \\
7,21 \pm 0,26 \\
5,83 \pm 0,60 \\
128,80 \pm 36,10 \\
267,00 \pm 11,60 \\
155,30 \pm 34,00 \\
58,10 \pm 22,00 \\
35,60 \pm 34,60\end{array}$ & $\begin{array}{r}3,2 \\
36,9 \\
33,0 \\
6,3 \\
3,1 \\
3,6 \\
10,3 \\
28,0 \\
4,3 \\
21,9 \\
37,9 \\
97,2 \\
\end{array}$ & $\begin{array}{r}120,83 \pm \\
3,58 \pm \\
7,87 \pm \\
4,43 \pm \\
2,60 \pm \\
4,79 \pm \\
8,31 \pm \\
65,00 \pm \\
224,00 \pm \\
\pm \\
= \\
4,11 \pm\end{array}$ & $\begin{array}{r}3,59 \\
0,15 \\
0,18 \\
0,07 \\
0,08 \\
0,17 \\
0,52 \\
1,04 \\
15,29\end{array}$ & $\begin{array}{l}3,0 \\
4,2 \\
2,3 \\
1,6 \\
3,1 \\
3,5 \\
6,3 \\
1,6 \\
6,8 \\
= \\
\overline{9,2}\end{array}$ & $\begin{array}{r}12 \\
12 \\
12 \\
12 \\
11 \\
12 \\
12 \\
12 \\
9 \\
\frac{-}{12}\end{array}$ & $\begin{array}{l}a>b \\
a>b \\
a>b \\
a \geq b \\
a>b \\
a>b \\
a=b \\
a>b \\
a=b \\
= \\
=\end{array}$ \\
\hline
\end{tabular}

Tab. 7

Mittelwerte und Standardabweichung von im SMA 12 und mit ,konventioneller" Methode analyslerten nicht ausgewalliten Patientenseren Kursiv gedruckte Testquotienten keninzeichinen zufallige Unterschlede zwisclien den zwei "Analysenverfahren". Bel Testquoticnten $\geq 2,0$ spreclien $0,05>p>0,01$ für den Unterschied. Bei Testquotienten $>2,6$ ist der gepriifte Unterschied signifikant (p $\leq 0,01$ )

\begin{tabular}{|c|c|c|c|c|c|c|}
\hline & & $\begin{array}{l}\text { Mittelwert } \\
\text { Anzahl der } \\
\text { Wertepaare }\end{array}$ & $\begin{array}{c}\text { andardabwelchung } \\
\text { SMA } 12(y) \\
\times \pm \sigma\end{array}$ & $\begin{array}{l}\text { Verglelchismethode (x) } \\
\qquad \pm 0\end{array}$ & & Testquotlent \\
\hline $\begin{array}{l}\text { Cholesterin } \\
\text { Calciun } \\
\text { Pliosphor } \\
\text { Bllirubln } \\
\text { Albumin } \\
\text { Gesamt-Eiwelß } \\
\text { Harnsture } \\
\text { Harnstoff } \\
\text { Glukose } \\
\text { Kreatinin } \\
\text { alk. Phospliatase } \\
\text { Lactatdehydrogenase } \\
\text { Aspartat-Transaminase }\end{array}$ & $\begin{array}{l}\mathrm{mg} / 100 \mathrm{ml} \\
\mathrm{mVal} / \mathrm{l} \\
\mathrm{mg} / 100 \mathrm{ml} \\
\mathrm{mg} / 100 \mathrm{ml} \\
\mathrm{g} / 100 \mathrm{ml} \\
\mathrm{g} / 100 \mathrm{ml} \\
\mathrm{mg} / 100 \mathrm{ml} \\
\mathrm{mg} / 100 \mathrm{ml} \\
\mathrm{mg} / 100 \mathrm{ml} \\
\mathrm{mg} / 100 \mathrm{ml} \\
\mathrm{K} / \mathrm{A}-\mathrm{E} \\
\text { WE } \\
\mathrm{KE}\end{array}$ & $\begin{array}{r}.100 \\
266 \\
218 \\
120 \\
87 \\
87 \\
216 \\
223 \\
74 \\
51 \\
112 \\
152 \\
97\end{array}$ & 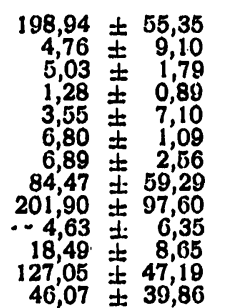 & 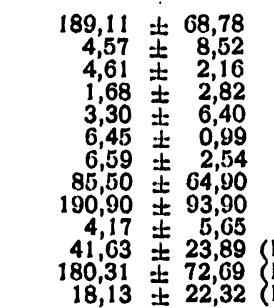 & (1. E. E. & $\begin{array}{l}1,11 \\
2,58 \\
2,20 \\
\left.7,48^{*}\right) \\
2,42 \\
2,23 \\
1,21 \\
7,75 \\
0,70 \\
0,38 \\
9,63 \\
7,07 \\
4,68\end{array}$ \\
\hline
\end{tabular}

*) Im parameterfrelen Vorzelchentest ermlttelt

Z. klin. Chem. u. klin. Biochem. / 7. Jahrg. 1969/ Heft 1 
Tab. 8

Beziehungen zwischen SMA 12- und bisherigen Analysenresultaten

\begin{tabular}{|c|c|c|c|c|}
\hline \multirow[t]{2}{*}{ Methode } & \multicolumn{2}{|c|}{ Korrelationskoeffizient $\mathbf{r}$} & \multicolumn{2}{|c|}{$\begin{array}{l}\text { Regressionsgerade } \\
y=b x+a\end{array}$} \\
\hline & $\mathbf{r} \pm \sigma_{\mathbf{r}}$ & $\mathrm{n}$ & & \\
\hline $\begin{array}{l}\text { Calcium } \\
\text { Phosphat } \\
\text { Gesamt-Eiweiß } \\
\text { Albumin } \\
\text { Alk. Phosphatase } \\
\text { Lactatdehydrogenase } \\
\text { Aspartat-Transaminase }\end{array}$ & $\begin{array}{l}0,7260 \pm 0,042 \\
0,8457 \pm 0,011 \\
0,8632 \pm 0,055 \\
0,9058 \pm 0,046 \\
0,7375 \pm 0,064 \\
0,6731 \pm 0,060 \\
0,7185 \pm 0,071\end{array}$ & $\begin{array}{r}266 \\
218 \\
87 \\
87 \\
112 \\
152 \\
97\end{array}$ & $\begin{array}{r}1,13 \\
1,85 \\
1,11 \\
0,35 \\
7,38 \\
48,26 \\
38,77\end{array}$ & $\begin{array}{l}0,7966 \\
0,6928 \\
0,9014 \\
0,9612 \\
0,2669 \\
0,4370 \\
0,4024\end{array}$ \\
\hline
\end{tabular}

Tab. 9

F-Prüfung der Wiederhol- und Vergleichsstreubereiche für Standardund Sammelserum in der Betriebsphase des SMA 12

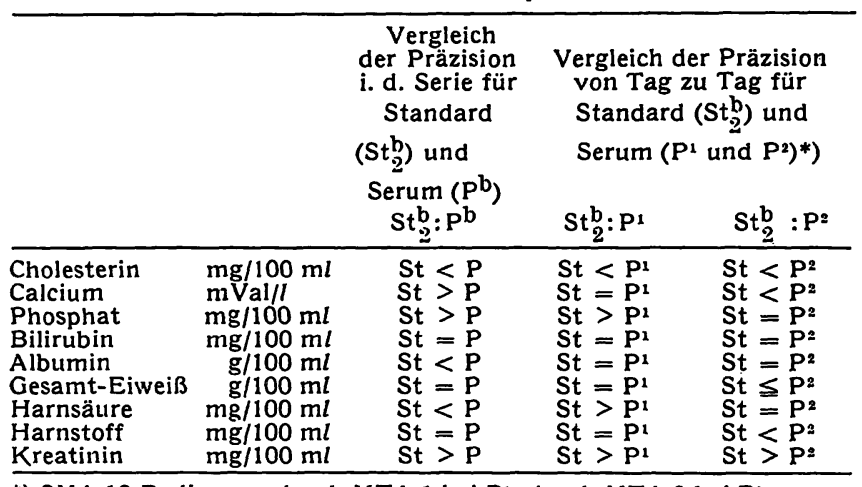

*) SMA 12 Befienung durch MTA 1 bei $P^{1}$, durch MTA 2 bei $P^{2}$

- am Wiederholstreubereich $(\mathrm{k}(\mathrm{P}) \times \sigma)$ gemessen - im SMA 12 günstiger ab (Tab. 1, b/c, Tab. 2, b/c). Die Variationskoeffizienten für serielle Cholesterin-, Kreatinin-, Glucose-, Lactatdehydrogenase-, Aspartattransaminase- und alkalische Phosphatasebestimmungen im SMA 12 unterscheiden sich nicht von denen bisheriger Methoden (Tab. 1, b und a, Tab. 2, a/c, b/c).

Vergleichswerte für Bilirubin und Albumin fehlen, Ausnahmen bilden Harnsäure und Gesamteiweiß. Der Variationskoeffizient für die „Eiweißbestimmung in der Serie" im SMA 12 stellt mit 1,4\% (für Standard, Tab. 3, b) bzw. 1,5\% (für Poolserum, Tab. 1, b) noch keine Belastung für die Präzision von Tag zu Tag (Tab. 4, a) dar, wohl aber ein $\mathrm{V}$ von $1,8 \%$ (Standard) bzw. 3,9\% (Poolserum) bei der Harnsäure, die mit anderem Nachweisprinzip im 2-Kanal-Autoanalyzer genauer gemessen wird (Tab. 1, c, 2, b/c, 3, b, 4 und 5, $\mathrm{b} / \mathrm{c}$ ).

2. Reproduzierbarkeit

a) Die Präzision von Tag zu Tag (Reproduzierbarkeit, reproducibility, Vergleichsstreubereich) für die ,,in der Routine" verwendeten SMA 12-Meßwerte wurde an Standardsera und einem gespeicherten Sammelserum von 2 unterschiedlich mit Laboratoriumsarbeit belasteten medizinisch-technischen Assistentinnen (MTA) kontrolliert (Tab. 4).

MTA 1 konzentrierte ihre volle Arbeitskapazität und außergewöhnliçhe Erfahrung auf die Wartung und Bedienung des SMA 12. Ihre über 14 Tage verteilten Vergleichsmessungen von Cholesterin, Calcium, Phosphor, Albumin, Gesamteiweiß, Harnsäure, und Harnstoff in Poolserum sind mit einem Variationskoeffizienten $\leqq 3,9 \%$ oder mit einem Vergleichsstreubereich $(2,57 \times \sigma)$ von weniger als $10 \%$ belastet. Kreatinin streut (bei einem V zwischen $4,2-5,0 \%$ ) zwischen $10-13 \%$ (Tab. .4, a).
Der Streubereich für Serumbilirubinbestimmungen entspricht nicht den praktischen Erfahrungen, Kontrolle mit stabilen und höheren Bilirubinkonzentrationen (vgl. Tab. 6, a/b) liefern bessere Resultate.

Die von dieser MTA in Poolserum erreichten Vergleichsstreubereiche für die SMA 12-Analysen sind entweder besser oder so gut wie die von konventionellen Bestimmungen (Tab. 5, a/c). Günstiger liegen Cholesterin, Phosphor, Harnsäure und Harnstoff, während der Vergleichsstreubereich von flammenphotometrisch bestimmteın Calcium, manuell erstelltem Kreatinin, für im 4-Kanal-Autoanalyzer gemessenes Kreatinin, für Harnstoff und Harnsäure gleich groß ist wie für die entsprechenden SMA 12-Analysen.

b) MTA 2 (Tab. 4, b) erreicht bei mehrseitiger Belastung und ungünstigeren Wartungsbedingungen mit dem SMA 12 im Poolserum für Phosphor, Albumin, Gesamteiweiß, Harnstoff und Kreatinin einen $\mathrm{V} \leqq 5,8 \%$ für Calcium und Cholesterin einen $\mathrm{V} \leqq 7,8 \%$. FürBilirubin und Harnsäure liegen die V-Werte sogar $\geqq 10 \%$.

Das bedeutet für die SMA 12-Messung von Serumphosphat, -albumin, -gesamteiweiß, -harnstoff und -kreatinin einen Vergleichsștreubereich von $\pm 15 \%$, für Cholesterin und Calcium bis 20\% (!) und mit den konventionellen Meßverfahren verglichen, eine nicht vertretbare Präzisionseinbuße bei der Calcium-, Harnsäureund Harnstoffmessung (Tab. 4, b und c, Tab. 5, b/c). Unter gleich ungünstigen Bedingungen erreicht nach einer Einarbeitungszeit diese MTA in Standardsera für Cholesterin, Phosphor, Bilirubin, Albumin, Gesamteiweiß und Harnstoff einen Vergleichsstreubereich $(2,57 \times s)$ von weniger als $10 \%$, also einen $V \leqq 3,9 \%$. Für Calcium muß mit einem $V$ von $4,2 \%$, für Harnsäure mit 6,3\%, für Glucose mit 6,8\% und für Kreatinin mit einem $V$ von $9,2 \%$ gerechnet werden (Tab. $6, \mathrm{~b}$ ).

c) Die von dieser MTA unter ungünstigen Bedingungen für SMA 12-Analysen gefundenen Schwankungen von Tag zu Tag liegen damit - teilweise im Gegensatz zu den unter optimalen Meßbedingungen gefundenen Ergebnissen (vgl. 2, a u. Tab. 5, a/c) - nicht besser, aber auch nicht schlechter als bei manueller Serumaufarbeitung und -messung (Tab. 5, b/c).

\section{Vergleich SMA 12 - Manuelle Methoden}

Dem oben Gesagten entspricht der Paarvergleich einer Stichprobe, die im SMA 12, und mit bisher üblichem Verfahren analysiert wurde. Die im SMA 12 bestimmten Cholesterin-, Bilirubin-, Glucose-, Kreatinin-, Harnstoff- und Harnsäurewerte unterscheiden sich nicht von mit manueller Analysenaufbereitung nach WATSON (19) oder nach Schoenheimer $(20,21)$ bzw. SpERRY (22) bestimmtem Cholesterin, nicht von "manuell“" ermitteltem Bilirubin (23) und nicht von im 1- oder 2-Kanal-Autoanalyzer bestimmten Kreatinin, Harnstoff, Harnsäure und Glucose. Zwischen „SMA 12“Phosphat-, -Calcium-, -Gesamteiweiß- und -Albuminund den im 2-Kanal-Autoanalyzer bestimmten Phosphatwerten, flammenphotometrisch gemessenem Calcium, dem „manuell“ bestimmten Gesamteiweiß und 
den aus der elektrophoretischen Eiweißverteilung errechneten Albuminkonzentrationen bestehen ebenfalls keine Unterschiede, wenn man die Signifikanzschranke auf $\mathrm{P}>99 \%$ oder einen Testquotienten von $=2,64$ ( $n=70$ ) festlegt (Tab. 7). Bei noch größerem Stichprobenumfang sind für diese Messungen allerdings Differenzen möglich. Die Korrelationskoeffizienten und Regressionsfunktionen zwischen den im SMA 12 und mit bisherigen Methoden ermittelten Calcium-, Phosphor-, Albumin-, Gesamteiweiß-, Aspartat-Transaminase-, Lactatdehydrogenase- und alkalischen Phosphatasewerten sind deshalb in Tabelle 8 zusammengestellt. Keine Übereinstimmung besteht zwischen den im optischen Test bei fortlaufender Registrierung und den im SMA 12 erhaltenen Aktivitäten für Aspartat-Transaminase, alkalische Phosphatase und Lactatdehydrogenase. Das liegt an den unterschiedlichen Reaktionsbedingungen und an der Aktivitätsangabe in verschiedenen Einheiten (siehe oben), die nicht oder nur mit Vorbehalten umgerechnet werden können.

Aus diesem Grund haben wir den Aspartat-Transaminase-Kanal für eine Kreatininmessung umgerüstet und lassen die für die Bestimmung der alkalischen Phosphatase und für die Lactatdehydrogenase vorgesehenen Kanäle ungenützt.

\section{Vergleich der Präzision in Standard- und Poolsera}

An den noch relativ weiten Vergleichsstreubereichen für die SMA 12-Messungen von Calcium, Glucose und Kreatinin (die Enzyme bleiben aus den genannten Gründen unberücksichtigt) mag die geringe Stabilität dieser Substanzen im Standard- und Poolserum mit Schuld tragen, zumal, wenn das Untersuchungsmaterial wiederholt auf Raumtemperatur aufgewärmt und dann wieder auf $4^{\circ}$ abgekühlt wird. Über den Einfluß, dẹn die Qualität des Untersuchungsmaterials auf den Wiederholund Vergleichsstreubereich nimmt, unterrichtet die Tabelle 9. Lagerungsbedingte Konzentrationsänderungen wirken sich auf serielle Untersuchungen kaum oder nicht aus, weshalb für die Kontrolle der Präzision in der Serie Poolserum und Standardserum gleichermaßen geeignet sind.

Für die Ermittlung der Präzision von Tag z" Tag muß dagegen das Untersuchungsgut über 3-5 Wochen stabil bleiben. Das ist in richtig aufbereitetem „laboreigenem" Poolserum wie in Standardsera gewährleistet (Tab. $9 \mathrm{St}_{2}^{\mathrm{b}} / \mathrm{P}^{1}$ ). Jedoch scheint sich eine weniger sorgfältige Gerätewartung und Bedienung eher am Vergleichsstreubereich für Poolserumanalysen, weniger am Standard auszuwirken (Tab. $9 \mathrm{St}_{2}^{\mathrm{b}}: \mathrm{P}^{1} ; \mathrm{St}_{2}^{\mathrm{b}}: \mathrm{P}_{2}$ ). Aus dieser Abhängigkeit von einem „dauerhaften Standard“ erwachsen der Präzision von Tag zu Tag für relative Konzentrationsmessungen - wie sie in TechniconGeräten üblich sind - die wichtigste, weil häufigste Störung.

Das von der Lieferfirma für den SMA 12 empfohlene Arbeitsschema, die den Serumuntersuchungen vorausgehenden Vergleiche zwischen Tages- und Vortagsstandard sind unbedingt erforderlich und als Minimum anzusehen. Wir haben das Einstell- und Kontrollschema für den Routinebetrieb inzwischen erweitert (siehe oben), denn auch bei identischen Werten in der Serie - garantiert durch das Analysenprinzip und zwischengeschaltete Standards - bleibt die Präzision von Tag zu Tag kritisch. Hier ist man von einem exakt geeichten, stabilen, dem $\mathrm{zu}$ untersuchenden Material möglichst ähnlichen Standard, abhängig. Eine Gegenkontrolle mit getestetem Poolserum gehört $z u$ unserem Kontrollbzw. Einstellprogramm.

Möglichst geringe Wiederhol- und Vergleichsstreubereiche für die SMA-12-Daten sind in dem Maße vordringlich, in dem ein derartiges Gerät den Datenausstoß verzehnfacht, den klinisch-chemischen Befund für den Einzelfall verbreitert und die $\mathrm{Zahl}$ der damit untersuchten Patienten vergrößert (24). Auch die umfangreichen "Vorarbeiten" implizieren, abgesehen von Rentabilitätsgründen, möglichst viele Serumanalysen, also einen vielstündigen Lauf des SMA 12 soweit es Reagerzienvorrat und Gerätewartung zulassen.

\section{Literatur}

1. Thiers, R. E. und K. M. Oglesby, Clin. Chem. (New York) 10, 246 (1964). - 2. EgGsteIN, M., Regensburger ärztl. Fortbildung. In Vorbereitung. - 3. Eggstein, M., R. AllneR, E. KuhlmanN und W. KNODEL, Klin. Wschr. 44, 424 (1966). - 4. HuANG, Analyt. Chem. 33, 1405 (1965). - 5. KessLER, G. und M. Wolfman, Clin. Chem. (New York) 10, 686 (1964). - 6. Gombino, S. R. und H. Schrerber, Technicon Symposion, 1964, Vortrag Nr. 54. 7. Ness, A. T., H. C. Dickerson und J. V. Pastewka, Clin. chim Acta (Amsterdam) 12, 532 (1965). - 8. Weichselbaum, T. E., Amer. J. Clin. Pathol., 7, 40 (1946). - 9. LofḶAND, Technicon Automation Analytical, 1963, S. 356 zit.: Techricon SMA 12 Manual. - 10. MARSH, W. H., B. Fingerhut und H. Mruler, Clin. Chem. (New York) 11, 624 (1965). - 11. Marsh, W. H., B. Fingerhut und E. Kirsch, Clin. Chem. (New York) 5, 119 (1959). - 12. Hochelra, N. J. und S. Wernhouse, Analyt. biochem. 10, 304 (1965). - 13. HochelLA, N. J. und S. WeINhouse,
Analyt. biochem. 13, 322 (1965). - 14. WACKER, W. E. C. und L. E. Dorfman, J. Amer. Med. Ass. 181, 972 (1962). - 15. Morgenstern, S., M. OkLander, J. Auerbach, J. Kaufasan und B. KueIN, Clin. Chem. (New York) 12, 95 (1966). - 16. Karmen, A., J. Clin. Invest., 34, 126 (1955). - 17. Hughes, H. K., Analyt. Chem. 24, 1349 (1962). - 18. Doerffel, K., Beurteilung von Analysenverfahren und -ergebnissen. Springer, Berlin-Heidelberg-New York, J. F. Bergmann München (1965). 19. Watson, D., Clin. chim. Acta (Amsterdam) 5, 637 (1950). 20. Schoenhermer, R. und W. M. SperRx, J. biol. Chemistry 106, 745 (1934). - 21. SChoenheimer, R. und W. M. SPERRY, J. biol. Chemistry 110, 655 (1935). - 22. SpERRY, W. M. und M. WeBB, J. biol. Chemistry 187, 97 (1950). - 23. Jendrassik, L. und P. GROF, Biochem. Z. 297, 81 (1938). - 24. Bock, H. E. und M. EgGsTein; Dtsch. Med. Wschr. 93, 985 (1968).

Prof. Dr. M. Eggstein 74 Tübingen, Olfried-Müller-Str. 